\title{
A Stress-Criterion-Based Model for the Prediction of the Size of Wear Particles in Boundary Lubricated Contacts
}

\author{
Aydar Akchurin ${ }^{1,2}$ (1) $\cdot$ Rob Bosman ${ }^{2}$ Piet M. Lugt ${ }^{2,3}$
}

Received: 22 May 2016/Accepted: 12 October 2016/Published online: 20 October 2016

(C) The Author(s) 2016. This article is published with open access at Springerlink.com

\begin{abstract}
In this paper, the formulation and validation of a model for the prediction of the wear particles size in boundary lubrication is described. An efficient numerical model based on a well-established BEM formulation combined with a mechanical wear criterion was applied. The behavior of the model and particularly the influence of the initial surface roughness and load was explored. The model was validated using measurements of the wear particles formed in steel-steel and steel-brass contacts. In the case of steel-steel contact, a reasonable quantitative agreement was observed. In the case of steel-brass contact, formation of the brass transfer layer dominates the particles generation process. To include this effect, a layered material model was introduced.
\end{abstract}

Keywords Wear particles size $\cdot$ Simulation - Boundary element method

\section{List of symbols}

$x, y, \tilde{z} \quad$ Spatial coordinates, $[\mathrm{m}]$

$p(x, y), s(x, y) \quad$ Normal and tangential surface stresses, $[\mathrm{Pa}]$ $u(x, y) \quad$ Deflection due to pressure $p(x, y),[\mathrm{m}]$

Aydar Akchurin

a.akchurin@utwente.nl

1 Materials Innovation Institute (M2i), P.O. Box 5008, 2600 GA Delft, The Netherlands

2 Laboratory for Surface Technology and Tribology, Department of Engineering Technology, University of Twente, P.O. Box 217, 7500 AE Enschede, The Netherlands

3 SKF Engineering and Research Centre, Kelvinbaan 16, 3439 MT Nieuwegein, The Netherlands

\begin{tabular}{|c|c|}
\hline$E, E_{1}, E_{2}$ & $\begin{array}{l}\text { Young's modulus of the materials in } \\
\text { contact, }[\mathrm{Pa}]\end{array}$ \\
\hline$v, v_{1}, v_{2}$ & $\begin{array}{l}\text { Poisson's ratio of the cylinder and the } \\
\text { substrate, }[-]\end{array}$ \\
\hline$E^{\prime}$ & $\begin{array}{l}\text { Composite Young's modulus, }[\mathrm{Pa}] \\
\frac{2}{E^{\prime}}=\frac{1-v_{1}^{2}}{E_{1}}+\frac{1-v_{2}^{2}}{E_{2}}\end{array}$ \\
\hline$z(x, y)$ & Measured surface roughness, $[\mathrm{m}]$ \\
\hline$h_{s}(x, y)$ & $\begin{array}{l}\text { Separation distance between the bodies, } \\
{[\mathrm{m}]}\end{array}$ \\
\hline$F_{C}$ & Load carried by surface contacts, $[N]$ \\
\hline$A_{c}$ & Direct contact area, $\left[\mathrm{m}^{2}\right]$ \\
\hline$N_{x}, N_{y}, N_{z}$ & $\begin{array}{l}\text { Number of grid points in spatial directions } \\
{[-]}\end{array}$ \\
\hline$R$ & Radius of the cylinder, $[\mathrm{m}]$ \\
\hline$f_{C}^{b}$ & $\begin{array}{l}\text { Friction coefficient in boundary } \\
\text { lubrication, }[-]\end{array}$ \\
\hline$B$ & Width of the cylinder, $[\mathrm{m}]$ \\
\hline$H$ & Hardness, $[\mathrm{Pa}]$ \\
\hline$\sigma_{y}$ & Yield stresses, $[\mathrm{Pa}]$ \\
\hline$\sigma_{i j}$ & Subsurface stresses, $[\mathrm{Pa}]$ \\
\hline$R_{q}$ & $\begin{array}{l}\text { Surface roughness (standard deviation of } \\
\text { surface heights), }[\mathrm{m}]\end{array}$ \\
\hline
\end{tabular}

\section{Introduction}

Failure of equipment due to wear has led to considerable effort in understanding wear and in the development of predictive models for wear. For example, Meng and Ludema [1] have identified 182 equations for different types of wear. Among them were empirical relations, contact mechanics-based approaches and equations based on material failure mechanisms.

One of the most famous and frequently used wear equations was developed by Holm and Archard in 1953 [2]. 
The model considers adhesive wear and assumes the sliding spherical asperities to deform fully plastic. The wear volume formed in a sliding distance $s$ under the applied normal load $F$ equals to $V_{T}=k * F / H * s$. The coefficient $k$ is known as a wear coefficient and is frequently used to compare the degree of the wear resistance of systems [3, 4]. It can be regarded as the probability that a wear particle will be formed during contact. In general, this wear coefficient is estimated experimentally. Although Archard originally developed this equation to model adhesive wear, it is now widely used for modeling abrasive, fretting and other types of wear [5] as well.

In general, wear models can be empirical or based on physical phenomena occurring in the system. Empirical models typically fit the experimental data with algebraic equations of arbitrary form. Although they require less investment in the model development, a good predictive capability can be achieved only with a great number of experimental data. Physically based models use the physical properties of the system and physical phenomena to predict wear. By doing so, it is possible to significantly reduce the experimental data needed for the model development. These models can be classified in analytical, semianalytical and numerical methods. Most of them are used to predict the wear volume and resulting life-time, rather than focusing on the size of the resulting wear particles. On the other hand, the particles size is more relevant in some applications. For example, oxidation of grease thickener and base oil is accelerated by metal particles [6,7], the severity of this phenomenon depends on the total surface area of the particles and therefore on their size, shape and number. Another example is that an autoimmune reaction of the body is highly dependent on the size of formed wear fragments in artificial joint replacements [8, 9]. Also, the environmental impact of small particles is of increasing concern [10].

Analytical wear models often link global parameters, such as load, speed, temperature with the wear volume or the wear track depth [11-15]. Although they are applicable only in specific cases, these models are very useful for understanding the relationship between governing parameters such as hardness, load or sliding speed.

Among the numerical models, a large group is represented by finite element analysis (FEM) [16, 17]. The application of these models is restricted to the analysis of simplified problems, such as smooth surfaces, single asperities, due to the associated computational costs. The numerical models frequently incorporate Archard's law or its modifications using local contact stress distributions obtained by FEM [18-25]. A number of researchers also considered multi-scale FEM-MD (molecular dynamic approach) coupling in order to perform nanometer-scale wear simulations [26-28].
Semi-Analytical models combine numerical methods with analytical solutions to speed up the calculations. This approach is also applicable to the calculation of the subsurface stresses [29-31] and temperatures in the contact [32]. Boundary element methods (BEM) are widely spread in this group. These models are extensively used in simulations of the contact of rough surfaces [29,33-35] and rely on the assumption that the contacting bodies are semiinfinite.

Application of BEM to model wear may be performed in the same way as is done in FEM, for example, by using Archard's wear law locally [36, 37]. Alternatively, a particle-by-particle removal model can be built. This type of wear model requires the formulation of a material rupture criterion, which is used to indicate the regions of the wear volume generation. A number of criteria can be found in the literature, including critical accumulated dissipated energy [13], critical accumulated plastic strain [17], critical accumulated damage [38], critical von Mises stress [39] and their variants $[14,15,40,41]$.

Critical damage-based models are typically used to simulate fatigue type of wear, in which the failure criterion is based on a critical number of cycles and stress intensity. A combination of a local Archard wear equation and cumulative damage model (Palmgren-Miner) was used by Morales-Espejel et al. [42, 43] to simulate mild wear and to predict the generation of micro-pits. The model showed a good qualitative agreement with the experimental data in the prediction of the size of the pits [42], as well as in the topography evolution [44]. At the same time, this approach requires the assessment of the fatigue limits at various loads and it introduces additional parameters that need to be determined. In practice, these values are rather hard to determine using representative conditions. This approach is definitely required to simulate the evolution of the surfaces in time. However, in this paper, this time scale is not addressed since the objective here is to predict the particle generation until a steady-state situation has occurred. The objective is not to predict when the particles are generated.

In the current paper, the focus is rather on the running-in process of sliding contacts. The time scale of the problem is not relevant. The wear process is considered not to be time but only strain/stress driven [39]. Oila [45] studied the mechanisms of the material degradation due to wear in high carbon steels and concluded that the wear particle formation is associated with the so called plastically deformed regions beneath the surface. Based on this work, Nelias [46] proposed a running-in wear model, using the accumulated plastic strain criterion to distinguish these regions. Bosman et al. [39] employed the Von Mises yield criterion to identify the plastically deformed regions and simulated the wear particles generation. In addition, they combined a thermal and wear model [39, 47, 48]. They 
successfully applied the combined model to predict the transition from mild to severe wear, as well as the level of severe wear and running-in. It should be noted that the application of the stress-based criterion is computationally more efficient than the plastic strain-based criterion. A dense mesh is required which results in much longer calculation times for the plastic strains.

In the present paper, the attention will be given to steelsteel and steel-brass materials, frequently encountered in rolling element-cage contacts in bearings. A simplified version of the model developed by Bosman et al. [39] will be used here. An engineering semi-analytical model (BEM) for the simulation of wear particle formation in boundary lubrication will be formulated based on a critical Von Mises stress and validated using experimental measurements of the wear particles' size. The model can be combined with the Archard's wear law to estimate the number of generated particles, as discussed in the current paper.

\section{Materials and Methods}

\subsection{Experimental Procedures}

Experimental data on the wear particles size formed in boundary lubrication sliding contacts of steel-steel and steel-brass were taken from an accompanying publication by the authors [49].

In the current paper, several additional sliding tests of steel cylinder-brass disk contacts were performed to study the formation of a brass transfer layer and obtain its thickness (it was not measured in Ref. [49]). The tests were performed at the same conditions as in Ref. [49] and are described below. Experiments were conducted under boundary lubrication conditions with a polyalphaolefin oil (PAO) as a lubricant. The viscosity of the oil was $68 \mathrm{cSt}$ at $40{ }^{\circ} \mathrm{C}$. AISI 52100 steel cylinders with surface roughness (root mean square, $R_{q}$ ) of around $50 \mathrm{~nm}$ were worn against brass disks with approximately $760 \mathrm{~nm}$ roughness. The cylinder was stationary, while the disk was rotating. The radius of the wear track was $17 \mathrm{~mm}$. The radius of the cylinder was $2 \mathrm{~mm}$ and the width was $6 \mathrm{~mm}$. The normal load was taken to be $2.5,5$ or $10 \mathrm{~N}$ (corresponding to 88 , 130 and $175 \mathrm{MPa}$ ) under a constant temperature of $80^{\circ} \mathrm{C}$ and a sliding velocity of $0.01 \mathrm{~m} / \mathrm{s}$.

To obtain the thickness of the transfer layer formed during the steel-brass wear tests, indentation marks were placed across the contact line of a cylinder before the test. Subsequently, the roughness profile, including the dents, was measured. After the wear test, the profile measurement was taken again and the initial and worn profiles were matched using the indent marks. After the test, an increase in the initial height profile of the cylinder was observed due to the presence of the transfer layer. The thickness of the transfer layer was obtained by measuring the difference in the height between the worn and initial profiles.

The roughness measurement was taken using a Keyence VK9700 laser scanning microscope. Indentations and hardness measurements were taken using a LECO microhardness tester LM100AT.

\subsection{Simulation Parameters}

During the simulations, a cylinder-on-disk configuration was used, corresponding to the experimental conditions [49], as discussed in the previous section.

The material properties used in the simulations are given in Table 1. The wear of the cylinder was neglected since it is significantly harder than the counter surface and the measured wear volume was three orders of magnitude smaller than that of the steel disk. The yield stress was calculated from the hardness using the rule of thumb $H=$ $2.8 \sigma_{y}[46]$.

The measured surface data were used in the simulations (see Fig. 1) unless explicitly mentioned. The friction coefficient $(0.16$ for steel-steel and 0.3 for steel-brass contacts) used in the current study was taken from [49] and was considered constant throughout the simulation.

\subsection{Contact Model}

In the present model, the contacting bodies were assumed to be semi-infinite solids and both surfaces were assumed to be rough. In tribological systems running in boundary lubrication, the elastic properties near the surface may be significantly different from the bulk due to the presence of chemically/physically absorbed layers. However, as it was shown in [48], the thickness of the altered layers is relatively small and the presence of the layer can be neglected

Table 1 Properties of the materials

\begin{tabular}{llll}
\hline Property & Cylinder & Disk, steel & Disk, brass \\
\hline$E, \mathrm{GPa}$ & 210 & 210 & 88 \\
$v$ & 0.3 & 0.3 & 0.3 \\
$H, \mathrm{GPa}$ & 6 & 1.8 & 2.64 \\
$\sigma_{y}, \mathrm{GPa}$ & - & 0.64 & 0.94 \\
$R, \mathrm{~mm}$ & 2 & $\infty$ & $\infty$ \\
$R_{q}, \mathrm{~nm}$ & 50 & 610 & 760 \\
Skewness & -0.27 & -0.84 & -1 \\
Kurtosis & 8.6 & 4.46 & 4.94 \\
Slope & 0.22 & 0.28 & 0.15 \\
\hline
\end{tabular}



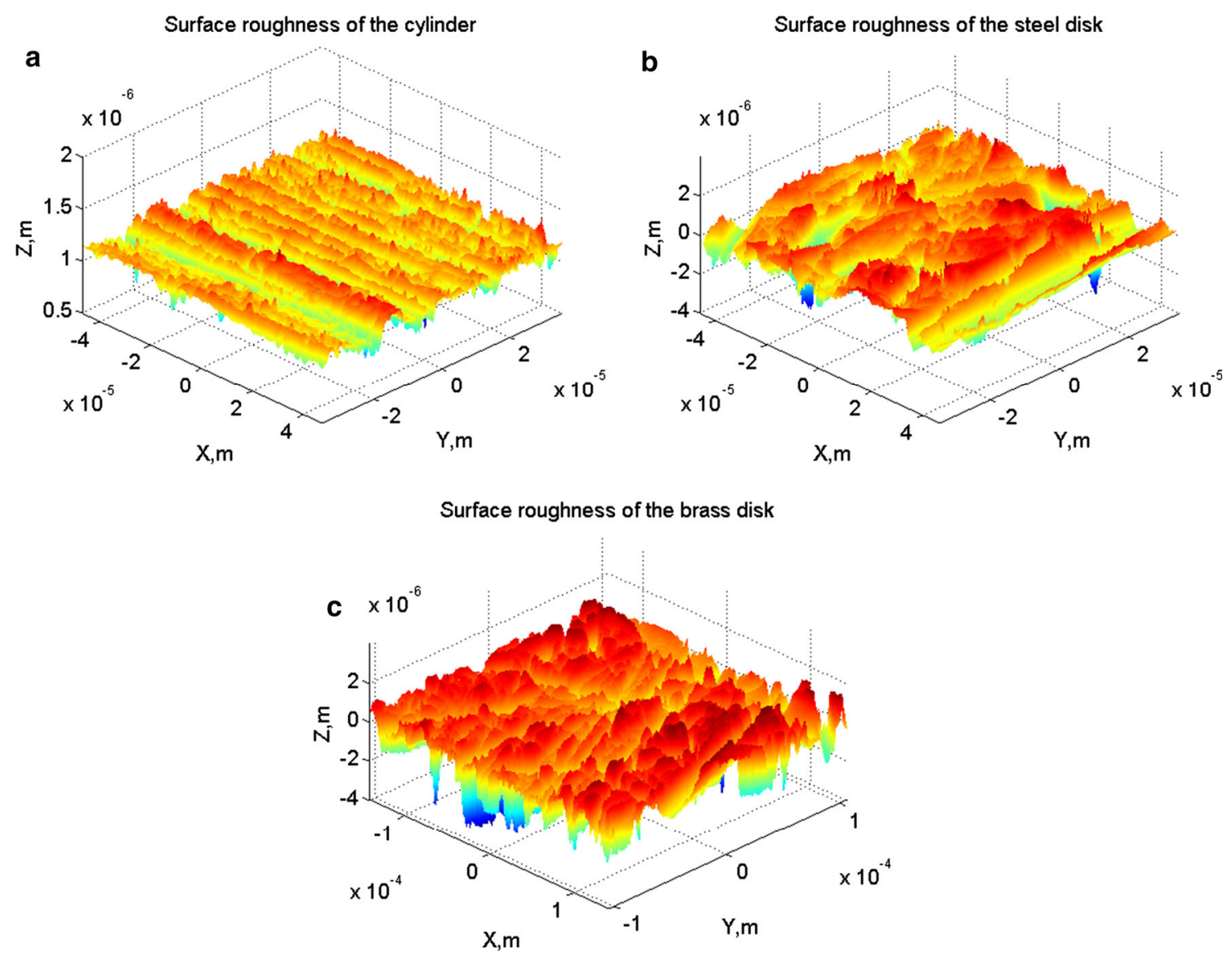

Fig. 1 Measured roughness profiles of the $\mathbf{a}$ cylinder, $\mathbf{b}$ steel and $\mathbf{c}$ brass disks

in the subsurface stress state calculation. Therefore, in this work, a homogeneous material model was assumed, unless explicitly mentioned.

To calculate the stresses on and beneath the surface, the half-space approximation was used. In this case, the contact pressure can be found by solving the following system of equations:

$$
\left\{\begin{array}{l}
u(x, y)=z(x, y)-h_{s}(x, y), \forall x, y \in A_{c} \\
p(x, y)>0, \forall x, y \in A_{c} \\
p(x, y) \leq H \\
F_{C}=\int p(x, y) \mathrm{d} x \mathrm{~d} y
\end{array},\right.
$$

where the deflection $u(x, y)$ can be calculated using the half-space approximation [50]. The solution method for these types of problems was developed by Polonsky and Keer [34] based on a single-loop "Conjugate Gradient Method." To reduce the computational burden only a fraction of the cylinder geometry in the direction perpendicular to sliding ( $y$ direction) was considered. Periodic boundary conditions were applied in this direction to mimic the full cylinder geometry.

Additionally, the computational time can be further decreased by implementing the discrete fast Fourier transformation technique (DC-FFT) [29], since the deflection $u(x, y)$ is a discrete convolution:

$u(x, y)=(K+S) \otimes p$,

where $K$ and $S$ are the influence matrices for normal and tangential stresses (tangential stress is $f_{c}^{b} \cdot p$ ). Equation (2) can be used for the calculation of the displacements in homogeneous [48], but also layered systems. For a layered body, expressions for $K+S$ are given in the frequency domain [51, 52].

The subsurface stress can be calculated using the following relation:

$$
\begin{aligned}
\sigma_{i j}(x, y, z)= & \iint p(\xi, \eta) F_{N i j}(x-\xi, y-\eta, z) \mathrm{d} \xi \mathrm{d} \eta \\
& +s(\xi, \eta) F_{S i j}(x-\xi, y-\eta, z) \mathrm{d} \xi \mathrm{d} \eta
\end{aligned}
$$

where $F_{N i j}$ and $F_{S i j}$ are the influence functions corresponding to the normal and tangential directions. Indices $(i, j) \in\{x, y, z\}$ are used to show the stress components. These integrals are also convolutions and can be calculated using the DC-FFT-based approach:

$\sigma_{i j}(x, y, z)=F_{N} \otimes p+F_{S} \otimes p$. 
Details are found in references [30, 53]. In case of layered surfaces, these functions are given in the frequency domain [51].

\subsection{Wear Particle Formation Criterion and Wear Model}

Once the subsurface stresses are obtained, the equivalent von Misses stresses can be calculated. The formation of a particle is then determined by the critical von Mises stress. More specifically, a particle will be formed, if in a certain volume of the body, the von Mises stress exceeds a critical value and if this volume is exposed to the surface. This is schematically shown in Fig. 2. The transition from elastic to elastic-plastic behavior is characterized by the yield stress [46, 53], which was therefore taken as a critical value, following Bosman et al. [39].

The flowchart of the wear model is shown in Fig. 3. After the initialization, the contact pressure and subsurface stresses are calculated. At this step, the grid resolution in vertical direction is refined 10 times by interpolation of the calculated von Mises stress to a refined grid using a polynomial function, for details see $[47,54]$. This keeps the computational time low while increasing the accuracy of the wear criterion application. Next, the particle formation criterion is applied. If this criterion is met, a particle is assumed to leave the contact. Subsequently, the surface profile is updated. The wear cycles continue until the equilibrium of the wear particle size has been reached.

\subsection{Surface Roughness Simulation}

The influence of the initial surface roughness on the wear particle's size was explored on artificially generated surfaces with different values for $R_{q}$, following the approach from $\mathrm{Hu}$ [55].

For each surface type, three surfaces were generated: $\mathrm{R}_{\mathrm{q}}=500, \mathrm{R}_{\mathrm{q}}=1000$ and $\mathrm{R}_{\mathrm{q}}=1500 \mathrm{~nm}$ for the softer surface and $\mathrm{R}_{\mathrm{q}}=50, \mathrm{R}_{\mathrm{q}}=500$ and $\mathrm{R}_{\mathrm{q}}=1500 \mathrm{~nm}$ for the harder surface.

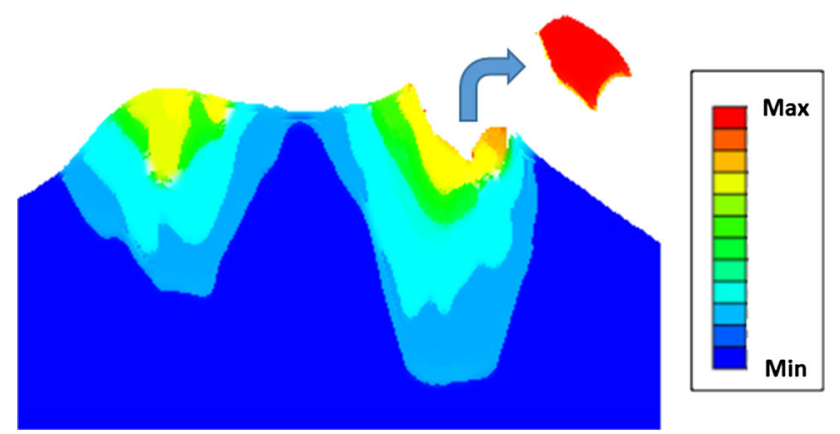

Fig. 2 Representation of the particle removal

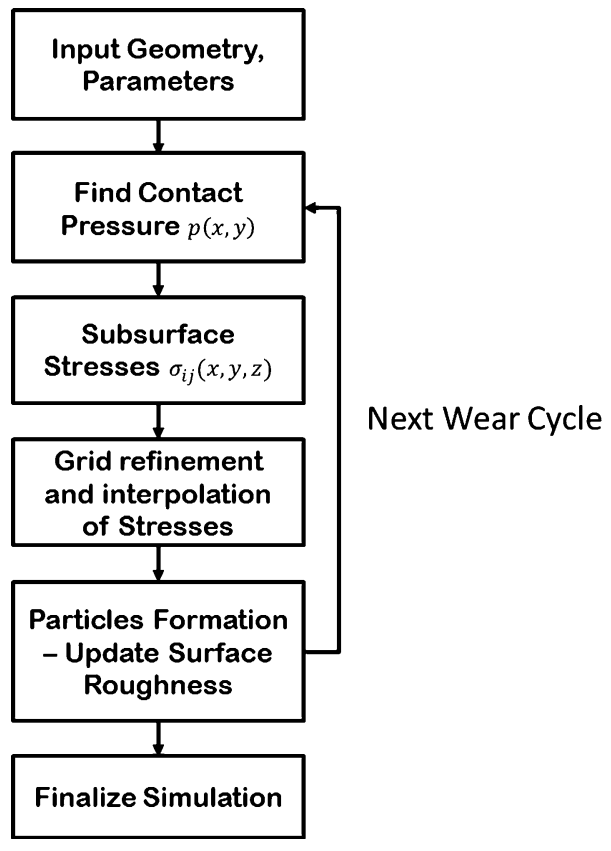

Fig. 3 Schematic diagram of the wear model

\section{Results and Discussion}

A set of simulations was performed to explore the numerical behavior and accuracy of the numerical scheme. To validate the model, the simulation results will be compared to the experimental data obtained in Ref. [49]. This includes dynamic light scattering (DLS) and atomic force microscope (AFM) measurement results. Equivalent radii of the particles were calculated to accommodate a direct comparison between the two methods (DLS determines only equivalent radii). This was calculated using:

$R_{i}=\left(\frac{V_{i}}{\pi} \frac{3}{4}\right)^{1 / 3}$,

where $R_{i}$ is the equivalent radius of the particle, and $V_{i}$ is its volume obtained from the simulation.

The simulations were done using homogenous material properties unless explicitly mentioned. The computational area was a square with a size of 4 times the Hertz contact radius.

\subsection{Numerical Convergence}

In this section, the convergence of the wear algorithm with respect to the particle's size is evaluated. Simplified simulations were performed by assuming that the steel disk is worn against a rigid flat surface using a normal load of $10 \mathrm{~N}$.

The computational grid size was gradually refined in lateral direction from $N_{x} \times N_{y} \times N_{z}=80 \times 80 \times 64$ data 
points (horizontal spacing $d_{x}=d_{y}=305, d_{z}=95 \mathrm{~nm}$ ) to $N_{x} \times N_{y} \times N_{z}=320 \times 320 \times 64 \quad\left(d_{x}=d_{y}=76 \mathrm{~nm}\right.$, $\mathrm{d}_{\mathrm{z}}=95 \mathrm{~nm}$ ). Variation of the average wear particle size is given in Fig. 4a (normalized to the value at the lowest grid). It can be seen that with the increase in the mesh density, the mean wear particle's size decreases and converges to a certain value. It does not change significantly with a further increase in the grid points.

In addition, the influence of the number of grid points in vertical direction on the results was studied. The number was gradually increased from $N_{z}=8\left(d_{z}=1500 \mathrm{~nm}\right)$ to $N_{z}=64\left(d_{z}=95 \mathrm{~nm}\right)$. The results are presented in Fig. $4 \mathrm{~b}$. As it was mentioned earlier, during the wear simulation, an interpolation is used in the $\mathrm{z}$ direction. Due to the interpolation, the influence of the initial depth resolution is decreased and the size of the particle does not depend on it, as shown in Fig. 4. More details in the description of the numerical algorithm are found in Ref. [54].

\subsection{General Simulation Results}

In this section, some typical simulation results are shown for a steel-steel contact, at a load of $10 \mathrm{~N}$ and a grid of $N_{x} \times N_{y} \times N_{z}=240 \times 240 \times 64$ points with $d_{x}=d_{y}=$ $102 \mathrm{~nm}$ and $d_{z}=95 \mathrm{~nm}$.

Figure 5a shows the variation of the wear particles size during the wear cycles. At the early stage of the wear process, large particles are formed followed by a decrease and stabilization of the particles size with further iterations. This behavior may be expected and can be explained by the evolution of the surfaces. Due to wear, the initial surfaces adapt and the stresses decrease, leading to a decrease in the number and size of the generated particles. This behavior, however, is not general and will depend on the initial surface roughness and the hardness of the material. If the initial surface is smooth, but the hardness of the material is low, the surface may become rougher due to large wear particles, leading to an increase in stress and further increase in the particles size until the equilibrium is reached.

The wear volume per iteration follows the same trend. In the beginning, the wear volume per iteration step is high. It results in a large wear volume in the first 50 cycles. Later, the wear volume per iteration stabilizes and the accumulation of the wear volume becomes slower.

As shown in Fig. 6a, the average contact pressure decreases only slightly during the wear process. This is due to a relatively low hardness of the disk. It restricts the local contacts from carrying high pressures, and most of the contact is in the full plastic regime. The evolution of the surface roughness is shown in Fig. 6b. In this particular case, the initially rough surface gets smoothened, as frequently reported, see for example $[44,56]$.

Based on the simulations of these cases, it can be concluded that some of the major characteristics of the wear process were captured. The running-in process is represented by the evolution of the surface profiles, wear volume, wear particles size and average contact pressure. In the following sections, the influence of the surface roughness and experimental validation of the model will be addressed.

\subsection{Wear Particles Size}

In this section, the results of the particles size simulation will be compared with the experimental data from Ref. [49], which is shown in Fig. 7. For the steel disks, the experiments show slightly higher values of the particles radius and a small increase with increasing load. The model predicts smaller particles and a somewhat higher increase. As discussed in Ref. [49], there is a bias of both AFM and DLS toward larger particles and this effect
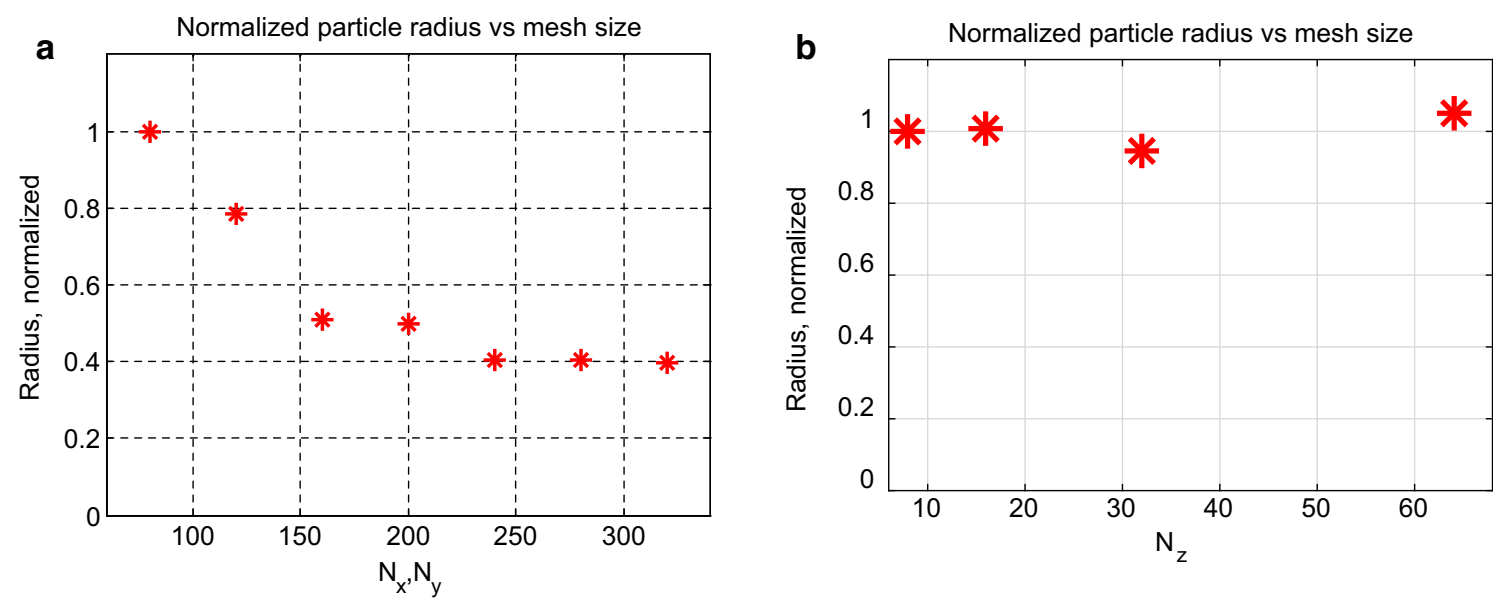

Fig. 4 Convergence of the wear particles size with the mesh size in lateral (a) and vertical (b) directions 

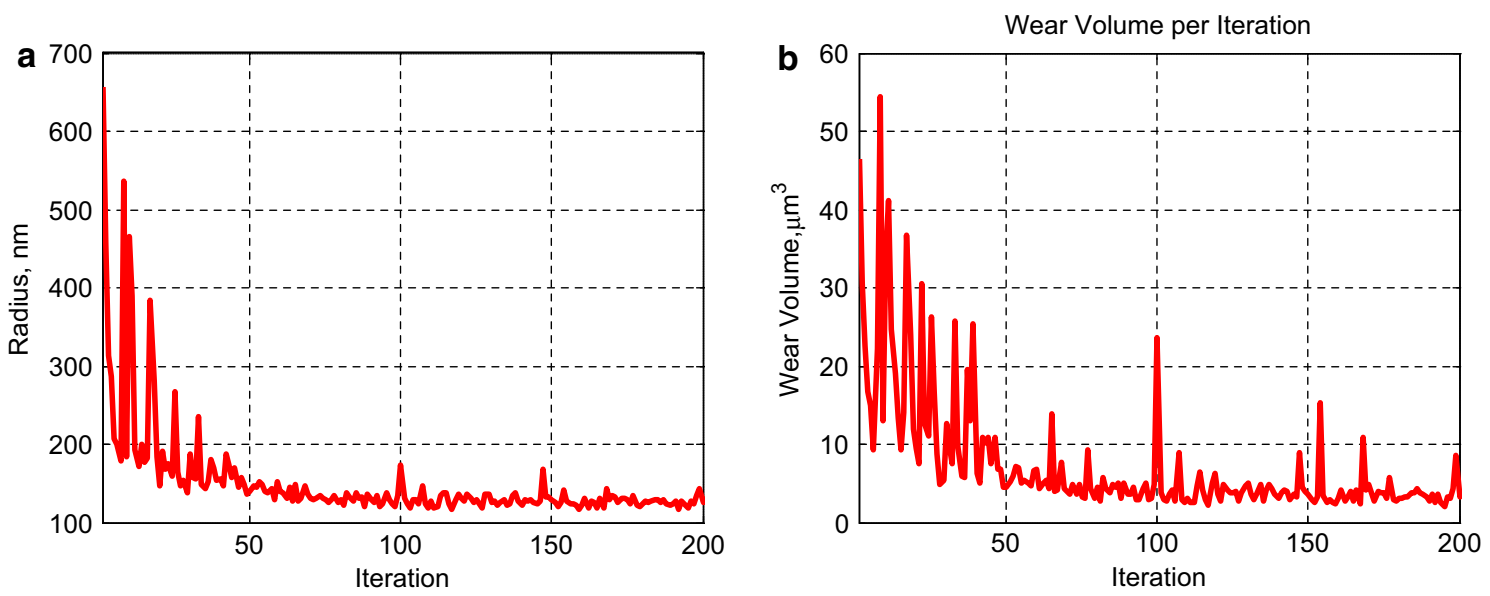

Fig. 5 Wear particles radius (a) and wear volume per iteration (b)
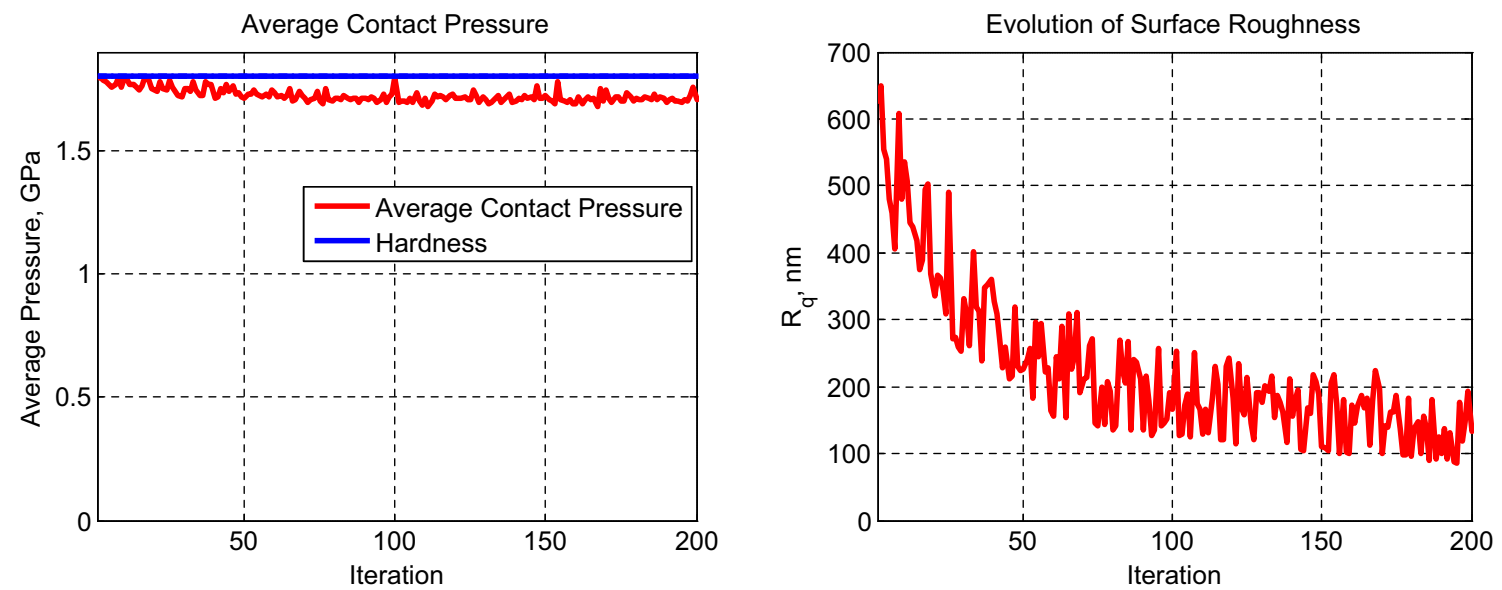

Fig. 6 Average contact pressure and surface roughness evolution
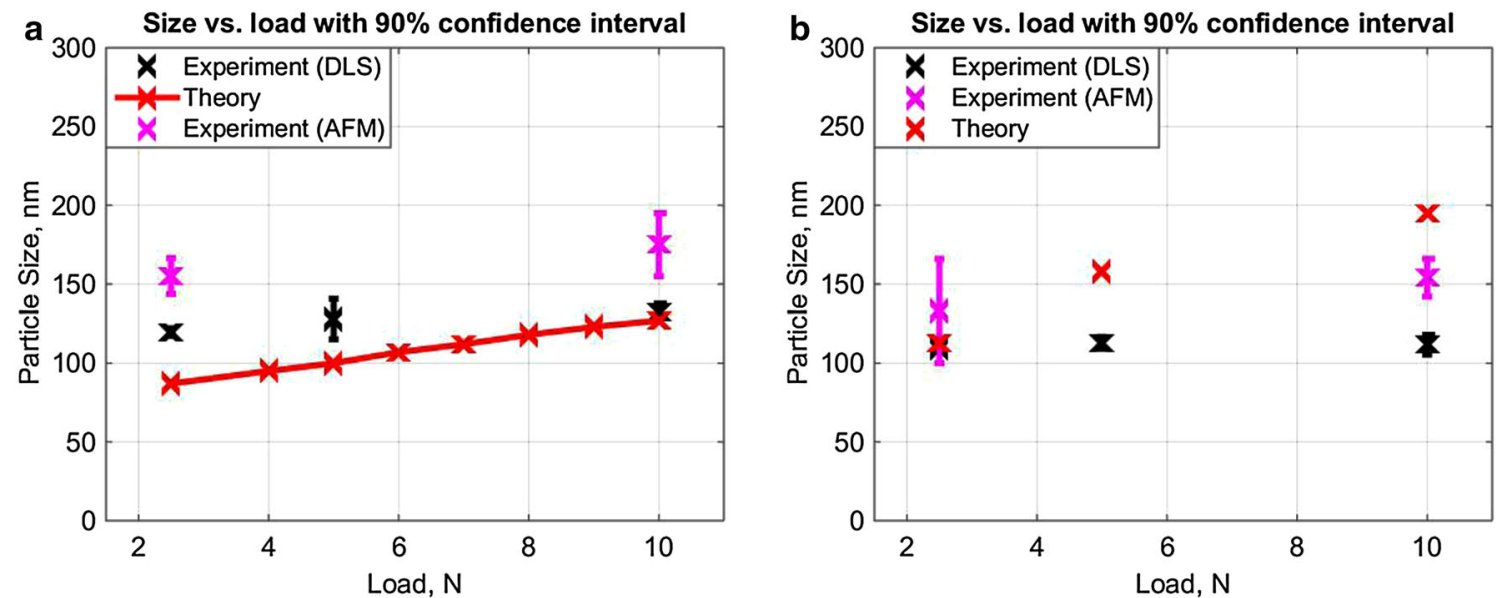

Fig. 7 Comparison of experimental data from Ref. [49] with theory for steel (a) and for brass (b). The wear particle size has been measured using $D L S$ (dynamic light scattering) and $A F M$ (atomic force microscopy) techniques. Equivalent radius is compared

becomes more important as the particles get smaller. The actual size of particles at all loads (and especially at lower loads, e.g., $2.5 \mathrm{~N}$ ) may be smaller than the experimentally obtained values. Therefore, the agreement between the simulations and the experiment can be considered as reasonable. 
In case of brass, both AFM and DLS do not show noticeable change in the particles size with load, in contrast with the model predictions. According to both DLS and AFM measurement, the brass particles have to be smaller than the steel particles, but the simulation shows an opposite behavior. In addition, the brass transfer layer was found on the surface of a cylinder after testing. It was therefore concluded that a different primary wear mechanism was encountered in the steel-brass contact, which will be discussed later.

Since the shape of the particles also plays an important role, comparison of the theoretical length, width and thickness with the AFM data from [49] was performed. The results for the steel-steel pair are shown in Table 2 and the steel-brass pair in Table 4. For steel-steel, the theoretical values are smaller, except for the thickness. It can also be noticed that the predicted variation with load of all three parameters is more pronounced than observed experimentally. This discrepancy increases with a decrease in the load, which may be linked to the limitations of the experimental data, as was discussed earlier [49].

\subsection{Influence of Surface Roughness on Wear Particles Size}

The influence of the initial surface roughness on the resultant wear particles size was explored using generated surfaces, and the result is shown in Fig. 8. It can be clearly seen that the variation of the surface roughness of the softer material does not influence the wear particles size, whereas the change in the topography of the harder surface produces a clear effect. This behavior was also observed experimentally [57].

\subsection{Brass Transfer Film and Wear of the Layered Surface}

In the case of steel-brass material combination, the presence of a brass transfer film was observed under an optical microscope, see Fig. 9. This layer was found to affect the size of the particles and resulted in a much larger deviation of the predicted and experimentally observed size.

Table 2 Specification of the particle's sizes, steel-steel contact

\begin{tabular}{llll}
\hline Property & AFM, [49] & Theory & Load, N \\
\hline Length, nm & 747 & 442 & 10 \\
Width, nm & 541 & 245 & 10 \\
Thickness, nm & 145 & 197 & 10 \\
Length, nm & 600 & 267 & 2.5 \\
Width, nm & 476 & 161 & 2.5 \\
Thickness, nm & 129 & 151 & 2.5 \\
\hline
\end{tabular}

Therefore, the initially proposed model cannot be applied to the steel-brass contact pair, and an improved model will be proposed in this section.

From Fig. 9 it can be seen that there is a sharp edge of the transfer film on the left side. This is not the case for the right, trailing, edge. By subtracting the surface profiles of the cylinder before and after the $10 \mathrm{~N}$ test, the thickness of the transfer film in the contact area was estimated to be in the range of $50-150 \mathrm{~nm}$, see Fig. 10. This figure also shows the accumulation of brass, probably generated by the wear particles in the inlet of the contact on the stationary surface. The inlet will be completely filled with the surplus of particles that do not fit in the contact. In the load carrying zone, the film is much thinner. Thin brass transfer films have been reported earlier in the literature. At 1-3 MPa contact pressure, a thickness of $<80 \mathrm{~nm}$ is mentioned in [56], and a thickness of $300-500 \mathrm{~nm}$ is reported in [58]. Regarding the test done with a $2.5 \mathrm{~N}$ load, it was clear that the thickness was smaller. Unfortunately, the accuracy of the measurement did not allow to get the exact value. The width of the film along the direction of sliding was found to be dependent on the applied normal load, as was also reported in [59].

The hardness of the brass film on the cylinder surface after the test was found to be close to the value of the hardness of the brass disk surface before the wear test (see Table 3), as also reported in the literature [60].

The formation of the brass transfer film is attributed to the strong adhesion of brass to the surface of the steel cylinder during sliding [59]. Hence, the wear particles are not formed by the mechanism that was modeled in the case of the steel-steel pair, i.e., direct particle removal. Once the (very thin) film is formed, the initial brass-steel contact is substituted by a brass-brass contact. A simulation with the present model showed that the predicted size of particles would be in the range of 500-600 nm (at a load of $10 \mathrm{~N}$ ), which is significantly larger than the experimental observation. However, the initial steel-brass contact is very quickly transformed into a brass against a transfer layer coated steel contact. This model was then employed in the simulation of the brass-against steel situation. The following assumptions were introduced:

1. The particles detached from the brass disk only form a transfer film and do not create loose wear particles.

2. Loose wear particles are only formed from the transferred layer with a given (uniform) thickness and based on the critical Von Mises stress criterion.

3. There is no return of the brass wear particles.

A schematic representation of the contact is shown in Fig. 11. In the load carrying area, the thickness of the layer was found to be in the range from 50 to $150 \mathrm{~nm}$. The 

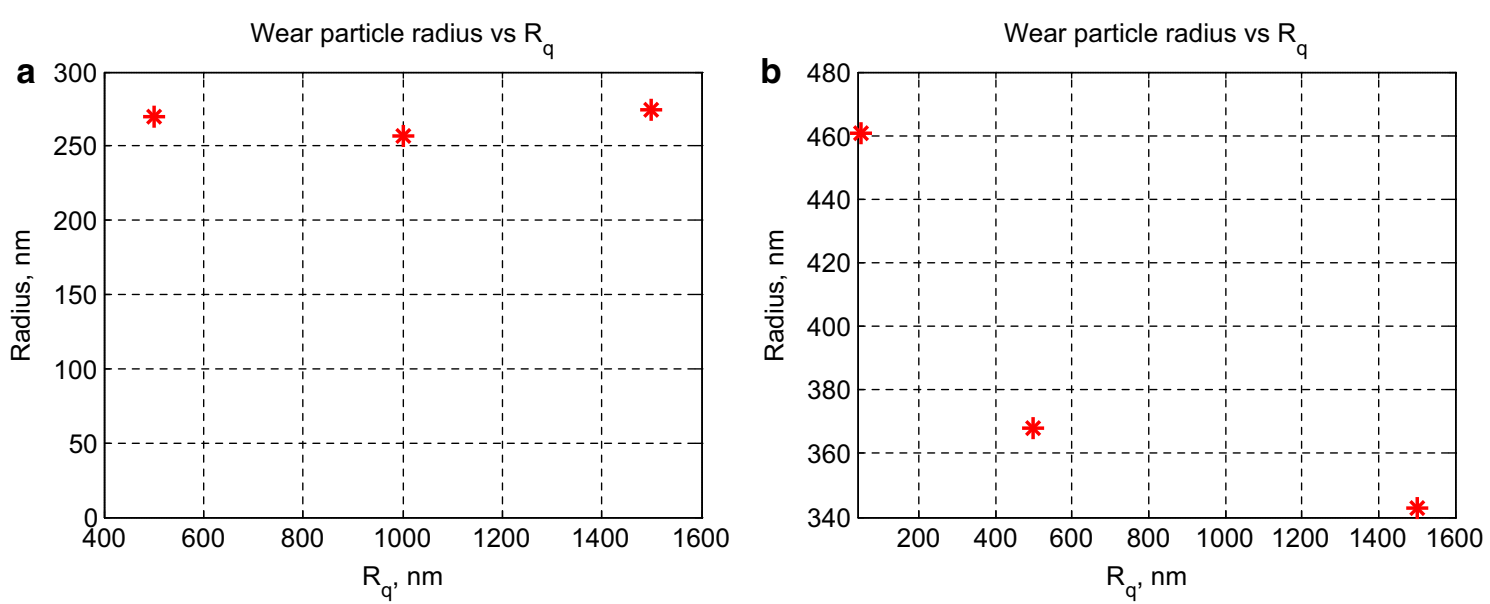

Fig. 8 Influence of the initial roughness of the softer (a) and harder (b) materials on the resultant particle size

Fig. 9 Microscope image of brass transfer film on the surface of the cylinder, $10 \mathrm{~N}$ load (a). Zoom in image (b)
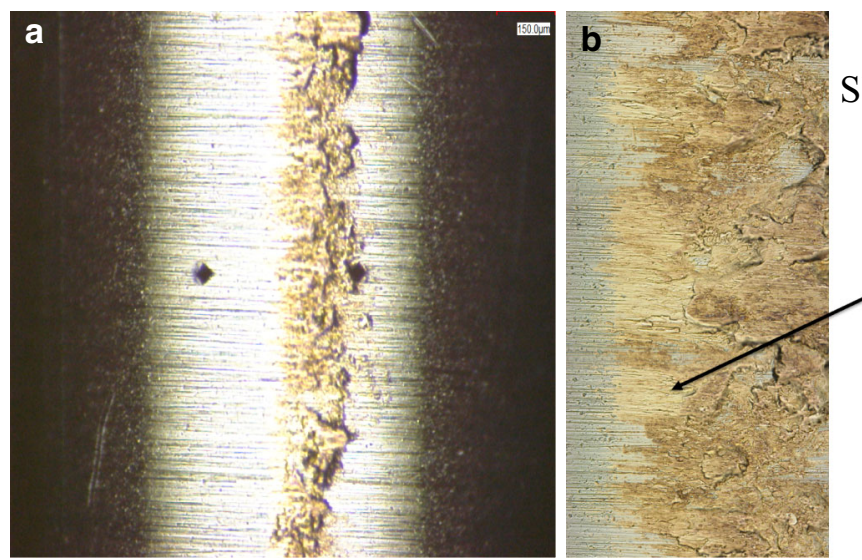

Sliding direction of the disk

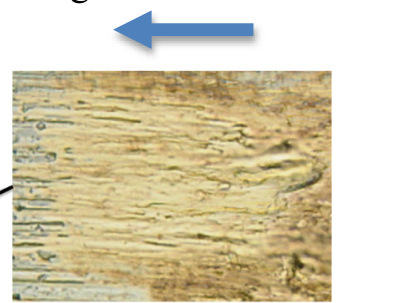

Fig. 10 Comparison of initial and after test cylinder surface profiles. The difference (pink color) indicates transferred or accumulated brass. The blue line represents the line perpendicular to the normal loading direction (Color figure online)

Table 3 Hardness of the brass

\begin{tabular}{ll}
\hline Hardness of the surface, GPa & 2.64 \\
Hardness of the transfer film, GPa & 2.96 \\
\hline
\end{tabular}

Transfer film hardness measured after 10-N load test

thickness of the layer was therefore taken to be equal to $100 \mathrm{~nm}$.

The results are shown in Fig. 12. In this figure, the initial model (Homogeneous Brass-Steel) is compared with the

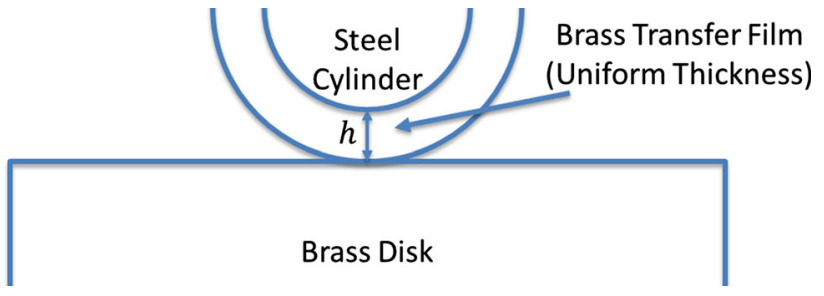

Brass Disk

Fig. 11 Schematic of the contact with brass transfer film

layered model (Brass-Brass Coated Steel) and experimental data. It can be seen that the introduction of the brass transfer layer considerably reduces the size of the wear particles below that of the steel particles corresponding to the experimental observations. In addition, the increase in the particles size with load is less pronounced for the layered model, in agreement with the experimental evidence.

Comparison of the predicted length, width and thickness of wear particles formed in steel-brass contact with the AFM data from Ref. [49] is shown in Table 4. The predicted values are always smaller, including the thickness. The variation of the thickness with load is limited in both simulation and experiment, which indicates that the 


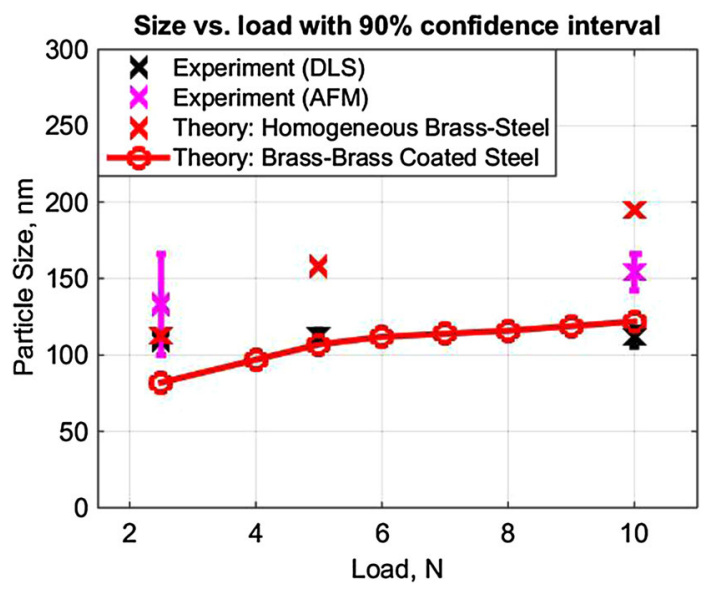

Fig. 12 Comparison of the model results and experiment for brass

Table 4 Specification of the particle's sizes, steel-brass contact

\begin{tabular}{llll}
\hline Property & AFM, [49] & Theory & Load, N \\
\hline Length, nm & 751 & 635 & 10 \\
Width, nm & 521 & 262 & 10 \\
Thickness, nm & 126 & 95 & 10 \\
Length, nm & 543 & 300 & 2.5 \\
Width, nm & 397 & 195 & 2.5 \\
Thickness, nm & 115 & 89 & 2.5 \\
\hline
\end{tabular}

thickness of particles is largely influenced by the thickness of the transfer layer $(50-150 \mathrm{~nm})$. As for the case with steel disk, the decrease in the size is less pronounced in the experimental data.

\subsection{Discussion}

The degradation of surfaces during the wear process is complex. It involves the influence of contact pressure, microstructural changes, material transformation, chemical reactions and material properties variation [45, 61].

The model described in this paper is a relatively simple method to predict the wear particles size distribution. If the removed volume is known, the size may also be used to calculate the number of wear particles resulting from the wear process. Unfortunately, the considered approach does not allow for the calculation of wear volume directly, due to the assumption of the immediate wear particle detachment if the critical stress is exceeded. In reality, the wear is a gradual, fatigue governed process and sometimes, even if the mentioned criterion is met, the particle will not detach, or if detached, stick to the surface again, and self-heal the surfaces. This leads to a several orders of magnitude overestimation of the wear volume. Therefore, to model the wear volume, at least an Archard's type of approach will be necessary to introduce the probability of wear particle detachment.

As it was shown in the case of a steel-steel contact, reasonable agreement is found between wear particles size prediction and measurements. In the steel-brass contacts, the dominant particle formation mechanism is different. The brass from the disk is first transferred to the counter body, forming a new sliding pair with different properties, and the loose wear particles are detached mainly from the transfer layer. Therefore, the particles formation is driven by the growth and rupture of this layer [62]. A better agreement with the experiment was found by considering the layered structure of the contacting bodies.

Employment of the BEM methods makes it possible to run a considerable number of iterations and to address the dynamics of the wear process, by including the running-in and steady-state regimes.

\section{Conclusions}

A simple wear algorithm based on subsurface stress calculation was introduced and validated using experimental data on the wear particles size. The particle generation criterion was based on a critical stress and geometrical boundary conditions. It was shown that the model works well for steel-steel contacts. In case of steel-brass contact, a transfer film was observed and it was concluded that the main wear particle formation mechanism is linked to the rupture of this layer. The model was therefore extended by including the influence of the transfer layer after which a better agreement was observed.

It was found that the resultant wear particles size is independent of the softer surface profile. In contrast, an increase in roughness of the harder material resulted in an increase in the wear particles detached from the softer material.

Overall, the performance of the model can be considered as satisfactory to predict the formation of wear particles both by direct removal as well as through formation and rupture of a transfer layer.

Acknowledgments The authors would like to thank SKF Engineering \& Research Center, Nieuwegein, The Netherlands, for providing technical and financial support. This research was carried out under Project Number M21.1.11450 in the framework of the Research Program of the Materials innovation institute M2i.

Open Access This article is distributed under the terms of the Creative Commons Attribution 4.0 International License (http://crea tivecommons.org/licenses/by/4.0/), which permits unrestricted use, distribution, and reproduction in any medium, provided you give appropriate credit to the original author(s) and the source, provide a link to the Creative Commons license, and indicate if changes were made. 


\section{References}

1. Meng, H.C., Ludema, K.C.: Wear Models and Predictive Equations: Their Form and Content. Elsevier, Amsterdam (1995)

2. Archard, J.F.: Contact and rubbing of flat surfaces. J. Appl. Phys. 24(8), 981-988 (1953). doi:10.1063/1.1721448

3. Ludema, K.: Friction, Wear, Lubrication: A Textbook in Tribology. CRC Press, Ann Arbor (1996)

4. Bhushan, B.: Principles and Applicaion of Tribology. Wiley, New York (1999)

5. Williams, J.A.: Wear modelling: analytical, computational and mapping: a continuum mechanics approach. Wear 225-229, 1-17 (1999)

6. Hurley, S., Cann, P.M., Spikes, H.A.: Lubrication and reflow properties of thermally aged greases. Tribol. Trans. 43(2), 221-228 (2008)

7. Jin, X.: The effect of contamination particles on lithium grease deterioration. Lubr. Sci. 7(3), 233 (1995)

8. MacQuarrie, R.A., Chen, Y.F., Coles, C., Anderson, G.I.: Wearparticle-induced osteoclast osteolysis: the role of particulates and mechanical strain. J. Biomed. Mater. Res. B Appl. Biomater. 69B, 104-112 (2004)

9. Green, T.R., Fisher, J., Stone, M., Wroblewski, B.M., Ingham, E.: polyethylene particles of a "critical size" are necessary for the induction of cytokines by macrophages in vitro. Biomaterials 19 , 2297-2302 (1998)

10. Olofsson, U., Olander, L., Jansson, A.: Towards a model for the number of airborne particles generated from a sliding contact. Wear 267, 2252-2256 (2009)

11. Argatov, I.I.: Asymptotic modeling of reciprocating sliding wear with application to local interwire contact. Wear 271(7-8), 1147-1155 (2011)

12. Mishina, H., Hase, A.: Wear equation for adhesive wear established through elementary process of wear. Wear $\mathbf{3 0 8}(1-2)$, 186-192 (2013). doi:10.1016/j.wear.2013.06.016

13. De Moerlooze, K., Al-Bender, F., Van Brussel, H.: A novel energy-based generic wear model at the asperity level. Wear 270(11-12), 760-770 (2011)

14. Huq, M.Z., Celis, J.P.: Expressing wear rate in sliding contacts based on dissipated energy. Wear 252(5-6), 375-383 (2002)

15. Aghdam, A.B., Khonsari, M.M.: On the correlation between wear and entropy in dry sliding contact. Wear 270(11-12), 781-790 (2011). doi:10.1016/j.wear.2011.01.034

16. Salib, J., Kligerman, Y., Etsion, I.: A model for potential adhesive wear particle at sliding inception of a spherical contact. Tribol. Lett. 30(3), 225-233 (2008)

17. Boher, C., Barrau, O., Gras, R., Rezai-Aria, F.: A wear model based on cumulative cyclic plastic straining. Wear 267(5-8), 1087-1094 (2009)

18. Molinari, J.F., Ortiz, M., Radovitzky, R., Repetto, E.A.: Finiteelement modeling of dry sliding wear in metals. Eng. Comput. 18(3/4), 592-609 (2001)

19. Podra, P., Andersson, S.: Simulating sliding wear with finite element method. Tribol. Int. 32(2), 71-81 (1999)

20. Hegadekatte, V., Huber, N., Kraft, O.: Modeling and simulation of wear in a pin on disc tribometer. Tribol. Lett. 24(1), 51-60 (2006)

21. Cruzado, A., Urchegui, M.A., Gomez, X.: Finite element modeling of fretting wear scars in the thin steel wires: application in crossed cylinder arrangements. Wear 318, 98-105 (2014)

22. Mattei, L., Di Puccio, F.: Influence of the wear partition factor on wear evolution modeling of sliding surfaces. Int. J. Mech. Sci. 99, 72-88 (2015)

23. Holmberg, K., Laukkanen, A., Turunen, E., Laitinen, T.: Wear resistance optimisation of composite coatings by computational microstructure modeling. Surf. Coat. Technol. 247, 1-13 (2014)
24. Holmberg, K., Laukkanen, A., Ghabchi, A., Rombouts, M., Turunen, E., Waudby, R., Suhonen, T., Valtonen, K., Sarlin, E.: Computational modelling based wear resistance analysis of thick composite coatings. Tribol. Int. 72, 13-30 (2014)

25. Tavoosi, H., Ziaei-Rad, S., Karimzadeh, F., Akbarzadeh, S.: Experimental and finite element simulation of wear in nanostructured NiAl coating. J. Tribol. 137, 014601 (2015)

26. Luan, B.Q., Hyun, S., Molinari, J.F., Bernstein, N., Robbins, M.O.: Multiscale modeling of two-dimensional contacts. Phys. Rev. E 74, 046710 (2006)

27. Xiao, S.P., Belytchko, T.: A bridging domain method for coupling continua with molecular dynamics. Comput. Methods Appl. Mech. Eng. 193, 1645-1669 (2004)

28. Crill, J.W., Ji, X., Irving, D.L., Brenner, D.W., Padgett, C.W.: Atomic and multi-scale modeling of non-equilibrium dynamics at metal-metal contacts. Model. Simul. Mater. Sci. Eng. 18, 034001 (2010)

29. Liu, S.: Thermomechanical Contact Analysis of Rough Bodies. Ph.D. thesis, Northwestern University (2001)

30. Bosman, R.: Mild Microscopic Wear Modeling in the Boundary Lubrication Regime. Ph.D. thesis, University of Twente (2011)

31. Leroux, J., Fulleringer, B., Nelias, D.: Contact analysis in presence of spherical inhomogeneities within a half-space. Int. J. Solids Struct. 47(22-23), 3034-3049 (2010)

32. Bos, J.: Frictional Heating of Tribological Contacts. Ph.D. thesis, University of Twente (1995)

33. Akchurin, A., Bosman, R., Lugt, P.M., van Drogen, M.: On a model for the prediction of the friction coefficient in mixed lubrication based on a load-sharing concept. Tribol. Lett. 59(1), 19-30 (2015)

34. Polonsky, I.A., Keer, L.M.: A numerical method for solving rough contact problems based on the multi-level multi-summation and conjugate gradient techniques. Wear 231, 206-219 (1999)

35. Tian, X., Bhushan, B.: A numerical three-dimensional model for the contact of rough surfaces by variational principle. J. Tribol. 118, 33-42 (1996)

36. Bosman, R., Schipper, D.J.: On the transition from mild to severe wear of lubricated, concentrated contacts: The IRG (OECD) transition diagram. Wear 269(7-8), 581-589 (2010)

37. Jerbi, H., Nelias, D., Baietto, M.-C.: Semi-analytical model for coated contacts. Fretting wear simulation. In Proceedings: LeedsLyon Symposium on Tribology, Lyon, France (2015)

38. Behesthi, A., Khonsari, M.M.: An engineering approach for the prediction of wear in mixed lubricated contacts. Wear 308, 121 (2013)

39. Bosman, R., Schipper, D.J.: Transition from mild to severe wear including running in effects. Wear 270(7-8), 472-478 (2011)

40. Ajayi, O.O., Lorenzo-Martin, C., Erck, R.A., Fenske, G.R.: Analytical predictive modeling of scuffing initiation in metallic materials in sliding contact. Wear 301, 57 (2013)

41. Jiang, J., Stott, F.H., Stack, M.M.: A Mathematical model for sliding wear of metals at elevated temperatures. Wear 181-183, 20 (1995)

42. Morales-Espejel, G.E., Brizmer, V.: Micropitting modelling in rolling-sliding contacts: application to rolling bearings. Tribol. Trans. 54, 625-643 (2011)

43. Brizmer, V., Pasaribu, H.R., Morales-Espejel, G.E.: Micropitting performance of oil additives in lubricated rolling contacts. Tribol. Trans. 56, 739-748 (2013)

44. Morales-Espejel, G.E., Brizmer, V., Piras, E.: Roughness evolution in mixed lubrication condition due to mild wear. Proc. Inst. Mech. Eng. Part J: J. Eng. Tribol. 229(11), 1330-1346 (2015)

45. Oila, A., Bull, S.K.: Phase transformations associated with micropitting in rolling/sliding contacts. J. Mater. Sci. 40, 4767-4774 (2005) 
46. Nelias, D., Boucly, V., Brunet, M.: Elastic-plastic contact between rough surfaces: proposal for a wear or running-in model. J. Tribol. 128, 236-244 (2006)

47. Bosman, R., Hol, J., Schipper, D.J.: Running in of metallic surfaces in the boundary lubrication regime. Wear 271(7-8), 1134-1146 (2011)

48. Bosman, R., Schipper, D.J.: Running-in of systems protected by additive rich oils. J. Tribol. Lett. 41, 263-282 (2011)

49. Akchurin, A., Bosman, R., Lugt, P.M.: Analysis of wear particles formed in boundary lubricated sliding contacts. Tribol. Lett. 63(2), 1-14 (2016)

50. Timoshenko, S.P., Goodier, J.N.: Theory of Elasticity, 3rd edn. McGraw-Hill, New York (1970)

51. Wang, Z.-J., Wang, W.-Z., Wang, H., Zhu, D., Hu, Y.-Z.: Partial slip contact analysis on three-dimensional elastic layered half space. J. Tribol. 132(2), 1-12 (2012)

52. Liu, S., Wang, Q.: Studying contact stress fields caused by surface tractions with a discrete convolution and fast Fourier transform algorithm. J. Tribol. 124(1), 36-45 (2002)

53. Jacq, C., Nelias, D., Lormand, G., Girodin, D.: Development of a three-dimensional semi-analytical elastic-plastic contact code. J. Tribol. 124, 653-667 (2002)

54. Boucly, V.: Contact and its Application to Asperity Collision, Wear and Running-in of Surfaces. INSA, Lyon (2008)
55. Hu, Y.Z., Tonder, K.: Simulation of 3-D random rough surface by 2-D digital filter and Fourier analysis. Int. J. Mach. Tools Manuf 32(1), 83-90 (1992)

56. Feser, T., Stoyanov, P., Mohr, F., Dienwiebel, M.: The running-in mechanisms of binary brass studied by in-situ topography measurements. Wear 303, 465-472 (2013)

57. Rowe, G.W., Kaliszer, H., Trmal, G., Cotter, A.: Running-in of plain bearings. Wear 34(1), 1-14 (1975)

58. Myshkin, N.K.: Friction transfer film formation in boundary lubrication. Wear 245, 116-124 (2000)

59. Kerridge, M., Lancaster, J.K.: The stages in a process of severe metallic wear. Proc. R. Soc. Lond. 236, 250-264 (1956)

60. Amirat, M., Zaidi, H., Djamai, A., Necib, D., Eyidi, D.: Influence of the gas environment on the transferred film of the brass $\left(\mathrm{Cu}_{64} \mathrm{Zn}_{36}\right) /$ steel AISI 1045 couple. Wear 267, 433-440 (2009)

61. Schofer, J., Rehbein, P., Stolz, U., Lohe, D., Zum Gahr, K.-H.: Formation of tribochemical films and white layers on self-mated bearing steel surfaces in boundary lubricated sliding contact. Wear 248, 7-15 (2001)

62. Nosovsky, M.: Entropy in tribology: in the search for applications. Entropy 12, 1345-1390 (2010) 